\title{
Signal Photon Flux and Background Noise in a Coupling Electromagnetic Detecting System for High Frequency Gravitational Waves (revised version)
}

\author{
F.Y. Li ${ }^{1, a} \quad$ N. Yang ${ }^{1, b} \quad$ Z.Y. Fang ${ }^{1, c}$ R.M.L. Baker Jr. ${ }^{2, d} \quad$ G.V. Stephenson ${ }^{3, e}$ \\ H. Wen ${ }^{1, f}$ \\ ${ }^{1}$ Department of Physics, Chongqing University, Chongqing 400044, P. R. China \\ ${ }^{2}$ GRAWAVE® LLC, 8123 Tuscany Avenue, Playa del Rey, California 90293, USA \\ ${ }^{3}$ Seculine Consulting, P0 Box 925, Redondo Beach, CA 90277, USA
}

\begin{abstract}
A coupling system between Gaussian type-microwave photon flux, static magnetic field and fractal membranes (or other equivalent microwave lenses) can be used to detect high-frequency gravitational waves (HFGWs) in the microwave band. We study the signal photon flux, background photon flux and the requisite minimal accumulation time of the signal in the coupling system. Unlike pure inverse Gertsenshtein effect (G-effect) caused by the HFGWs in the GHz band, the the electromagnetic (EM) detecting scheme (EDS) proposed by China and the US HFGW groups is based on the composite effect of the synchro-resonance effect and the inverse G-effect. Key parameters in the scheme include first-order perturbative photon flux (PPF) and not the second-order PPF; the distinguishable signal is the transverse first-order PPF and not the longitudinal PPF; the photon flux focused by the fractal membranes or other equivalent microwave lenses is not only the transverse first-order PPF but the total transverse photon flux, and these photon fluxes have different signal-to-noise ratios at the different receiving surfaces. Theoretical analysis and numerical estimation show that the requisite minimal accumulation time of the signal at the special receiving surfaces and in the background noise fluctuation would be $\sim 10^{3}-10^{5}$ seconds for the typical laboratory condition and parameters of $h_{\text {r.m.s. }} \sim 10^{-26}-10^{-30}$ at $5 \mathrm{GHz}$ with bandwidth $\sim 1 \mathrm{~Hz}$. In addition, we review the inverse G-effect in the EM detection of the HFGWs, and it is shown that the EM detecting scheme based only on the pure inverse G-effect in the laboratory condition would not be useful to detect HFGWs in the microwave band. PACS numbers: 04.30Nk, 04.25Nx, 04.30Db, 04.80Nn ${ }^{a}$ cqufangyuli@hotmail.com, ${ }^{b}$ cquyangnan@cqu.edu.cn ${ }^{c}$ zyf@cqu.edu.cn ${ }^{d}$ DrRobertBaker@GravWave.com ${ }^{e}$ E-mail: seculine@gmail.com ${ }^{f}$ wenhaowww@yahoo.com.cn
\end{abstract}




\section{Introduction.}

The first mention of high-frequency gravitational waves (HFGWs)was during a lecture in 1961 by Robert L. Forward[1]. The lecture was based upon a paper concerning the dynamics of gravity and Forward's work on the Weber Bar. The first actual publication concerning HFGWs was in mid 1962 when M.E Gertsenshtein[2] authored the pioneering paper entitled "wave resonance of light and gravitational waves" (it is often called Gertsenshtein effect). The next publication was in August of 1964 when L.Halpern and B.Laurent[3]; they suggested at some earlier stage of development of the universe (the big bang) conditions were suitable to produce strong relic gravitational radiation. They then discuss "short wavelength" or HFGWs and even suggest a 'laser' generator of HFGWs analogous to a laser for EM 'generation'. In 1968 R.A.Isaason authored papers [4,5] concerned with "Gravitational Radiation in the Limit of High Frequency". L.P.Grishchuk and M.V.Sazhin in the periods of 1974-1975 disccussed a scheme on "Emission of gravitational waves by an electromagnetic cavity and detection"[6,7], which also involved HFGWs. In 1974 G.F.Chapling, J.Nuckolls and L.L.Woods[8] suggested the generation of HFGWs by nuclear explosions and in 1978. V.B.Braginsky and V.N.Rudenko discussed detection and generation of the HFGWs [9]. In 1979 S.W.Hawking and W.Isreal[10] presented an actual definition for HFGWs having frequencies in excess $100 \mathrm{KHz}$. However, genuine attention to HFGWs was occurred from the 1990's for the following reasons:

(1)The maximal signal and peak of the relic GWs, expected by the quintessential inflationary models (QIM) [11-15] and some string cosmoogy scenarios[16-18], may be firmly localized in the GHz band, and their root-mean-square (r.m.s) values of the dimensionless amplitudes might reach up to $\sim 10^{-30}-10^{-33}$. Such works continue today.

(2) The thermal motion of plasma of stars, the interaction of the EM waves with interstellar plasma and magnetic fields, and the evaporation of primordial back holes[19], are possible means to generate the HFGWs.

(3)Study of nano-piezoelectiric resonator scheme[20], high-energy particle beam[21-25] and the construction of the LHC[26] are possible methods to produce HFGWs. Their frequencies may reach up to $10^{9} \mathrm{~Hz}$ and higher.

(4) Some HFGW detectors have already been constructed and more 
have been proposed. The constructed HFGW detectors include a toroidal waveguide scheme[27,28] and a coupled superconducting spherical cavities system[29,30]. Proposed detecting schemes include small laser interferometers detectors[31] and the coupling system of Gaussian beam, static magnetic and fractal membranes [32]. In Table 1 we list some possible HFGW sources and their major mechanisms.

In this paper our attention is focused on signal photon flux, the background photon flux(BPF) and their signal-to-noise ratios in the coupling EM detection scheme. We compute the signal photon fluxes, the signalto-noise ratios, and discuss displaying condition and the requisite minimal accumulation time of the signal in the background noise fluctuation. In addition, we review the inverse G-effect in the EM detection of the HFGWs. It is shown that the pure inverse G-effect in the laboratory condition cannot by itself detect the expected HFGWs, but the current EM detecting scheme might greatly improve detecting sensitivity and narrow the gap between the theoretical estimation of the expected HFGWs and the possibility of their detection.

The outline of this paper is the following; In Sec. I we present a brief history of the HFGWs research, including analyses of some possible HFGW sources. In sec. II we review the detecting scheme based on the pure inverse G-effect. In Sec.III we discuss the EM perturbation generated by the HFGW in coupling system between the static magnetic and the plane EM wave. In sec.IV we study the EM perturbative effect of the HFGW in the coupling system between the Gaussian typemicrowave photon flux, the static magnetic field, and the fractal membranes (or other equivalent microwave lenses), and give theoretical analysis, numerical estimations and a brief review to the role of the fractal membranes or other equivalent microwave lenses. Our conclusions are summarized in Sec V.

\section{Detecting scheme based on the inverse Gertsenshtein effect.}

It is well known that if an electromagnetic wave (EMW) propagates in a transverse homogeneous static magnetic field, it can generate the 
Table 1: Some possible HFGW sources and relevant paramenters

\begin{tabular}{|c|c|c|c|}
\hline Sources & Amplitude & Frequency & Characteristic \\
\hline $\begin{array}{l}\text { HFRGWs in } \\
\text { the quintessen- } \\
\text { tial inflation- } \\
\text { ary models } \\
\text { [11-15] }\end{array}$ & $h_{r m s} \sim 10^{-30}-10^{-32}$ & $v \sim 10^{9}-10^{10} \mathrm{~Hz}$ & Stochastic background \\
\hline $\begin{array}{l}\text { HFRGWs in } \\
\text { some string } \\
\text { cosmology } \\
\text { scenarios } \\
{[16-18]}\end{array}$ & $h_{r m s} \sim 10^{-30}-10^{-34}$ & $v \sim 10^{8}-10^{11} \mathrm{~Hz}$ & Stochastic background \\
\hline $\begin{array}{l}\text { Solar plasma } \\
\text { [19] }\end{array}$ & $h_{r m s} \sim 10^{-39}$ & $v \sim 10^{15} \mathrm{~Hz}$ & On the earth \\
\hline \begin{tabular}{l}
\multicolumn{3}{l}{ High-energy } \\
particles (e.g., \\
Fermi ring) \\
{$[24]$}
\end{tabular} & $h_{r m s} \sim 10^{-39}-10^{-4 I}$ & $v \sim 10^{4} \mathrm{~Hz}-10^{5} \mathrm{~Hz}$ & $\begin{array}{l}\text { On the center, the fre- } \\
\text { quency depends on the } \\
\text { rotating frequency of the } \\
\text { particles in the Fermi } \\
\text { ring }\end{array}$ \\
\hline $\begin{array}{l}\text { Stanford Lin- } \\
\text { ear Collider } \\
(\mathrm{SCL})[21]\end{array}$ & $h_{r m s} \sim 10^{-39}$ & $v \sim 10^{23} \mathrm{~Hz}$ & $\begin{array}{l}\text { On the collision cen- } \\
\text { ter, the frequency de- } \\
\text { pends on the self-energy } \\
\text { and the Lorenty factor of } \\
\text { high-energy } e^{+} e^{-} \text {beams }\end{array}$ \\
\hline $\begin{array}{lr}\text { The } & \text { Large } \\
\text { Hadron } & \text { Col- } \\
\text { lider } & \text { (LHC) } \\
{[26]} & \end{array}$ & & & $\begin{array}{l}\text { This is a continu- } \\
\text { ous spectra of high- } \\
\text { frequency gravitons, } \\
\text { only integrals for the } \\
\text { total spectra distribution } \\
\text { range might provide an } \\
\text { indirect effect. }\end{array}$ \\
\hline $\begin{array}{l}\text { Nano- } \\
\text { piezoelectric } \\
\text { crystal array } \\
(\text { size of } \\
100 \mathrm{~m})[20]\end{array}$ & $h_{r m s} \sim 10^{-28}-10^{-31}$ & $v \sim 10^{9}-10^{10} \mathrm{~Hz}$ & $\begin{array}{l}\text { On the wave zone, ef- } \\
\text { fective cross section of } \\
\text { the gravitational radia- } \\
\text { tion would be less than } \\
0.01 \mathrm{~m}^{2}\end{array}$ \\
\hline
\end{tabular}


gravitational wave (GW). This is just the G-effect[2]. Then converting probability of the EMW (photons) into the GW (graviton) is given by $[33,34]$ (in CGS units)

$$
P \approx 4 \pi G B^{2} L^{2} / c^{4},
$$

where $G$ is Newton's gravitational constant, $B$ is the static magnetic field. Contrarily, if a $\mathrm{GW}$ passes through a transverse homogeneous static magnetic field, then it can generate an EMW (photon flux), which propagates only in the same and in the opposite propagating directions of the GW. The latter is weaker than the former or is absent. This is just the pure inverse G-effect $[33,35]$. Whether the G-effect or its inverse effect, the conversion rate between the GWs (gravitons) and the EMWs (photons) is extremely low. For example, if $B=10 T=10^{5} \mathrm{Gauss}$, $L=10 \mathrm{~m}=1000 \mathrm{~cm}$, from Eq.(1), we have

$$
P \approx 1.0 \times 10^{-32} \text {. }
$$

For the EM perturbative effect caused by the GWs in the EM fields, one's attention is often focused to the inverse G-effect. In order to consider the pure inverse G-effect in the laboratory size, the wavelength of GWs should be the comparable with the laboratory dimension. Thus the high-frequency GWs (HFGWs) in the microwave band $\left(\sim 10^{8}-10^{10} \mathrm{~Hz}\right)$ would be suitable researching object. In fact, physical foundation of the G-effect is the Einstein-Maxwell equations in the weak field condition, while the physical foundation of the inverse G-effect is classical electrodynamics in curved spacetime. If a circular polarized HFGW passes through the transverse homogenous static magnetic field, according to the electrodynamical equations in curved spacetime, the EMW produced by the interaction of the HFGW with the static magnetic field can be given by $[32,35]$ (in order to compare possible experimental effect, from now, we use MKS units).

$$
\begin{gathered}
\vec{E}^{(1)} \approx A \hat{B}_{y}^{(0)} k_{g} c z \exp \left[i\left(k_{g} z-\omega_{g} t\right)\right], \\
\vec{B}^{(1)} \approx A \hat{B}_{y}^{(0)} k_{g} z \exp \left[i\left(k_{g} z-\omega_{g} t\right)\right],
\end{gathered}
$$

where $\vec{E}^{(1)}$ and $\vec{B}^{(1)}$ are parallel to the xy-plane and $\vec{E}^{(1)} \perp \vec{B}^{(1)}$. We also assume $A=A_{\oplus}=A_{\otimes}=\left|h_{\oplus}\right|=\left|h_{\otimes}\right|$, as the amplitudes of the HFGW with two polarization states, and the superscript (0) denotes the background EM fields, the notation ${ }^{\wedge}$ indicates the static EM fields, respectively. Here we neglected the EMW propagating along the negative direction of the 
Z-axis, because it is often much less than the EMW propagating along the positive direction of the z-axis. Eqs.(3) and (4) show that such perturbative EM fields have a space accumulation effect $(\propto z)$ in the interacting region: this is because the GWs (gravitons) and EMWs (photons) have the same propagating velocity in a vacuum, so that the two waves can generate an optimum coherent effect in the propagating direction $[33,35]$. From Eqs. (3) and (4), the power flux density of the EMW in the terminal receiving surface $(\mathrm{z}=\mathrm{L})$ will have maximum $(\mathrm{z}=\mathrm{L}$, see Figure 1)

$$
u_{e m}=1 / \mu_{0} \cdot\left|\vec{E}^{(1)} \times \vec{B}^{(1)}\right| \approx 1 / \mu_{0} \cdot\left(A \hat{B}_{y}^{(0)} k_{g} L\right)^{2} c .
$$
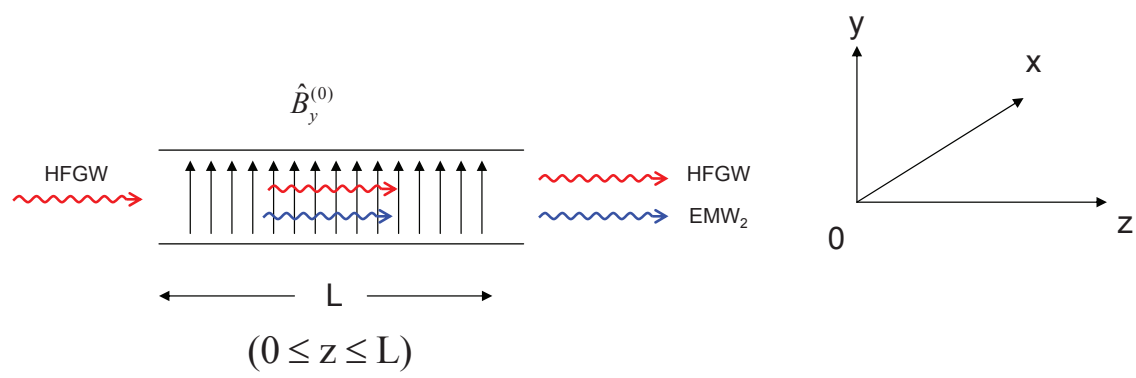

$(0 \leq \mathrm{z} \leq \mathrm{L})$

Figure 1: If a HFGW passes through a static magnetic field $\hat{\vec{B}}_{y}^{(0)}$, the interaction of the HFGW with the static magnetic field will produce an EMW, where $\mathrm{L}$ is the interacting dimension between the HFGW and the static magnetic field. The $\mathrm{EMW}_{2}$ has maximum in the terminal position $(\mathrm{Z}=\mathrm{L})$ of the interacting volume due to the space accumulation effect in the propagating direction (the z-direction).

In order to compare and analyze the EM perturbative effect under 
typical laboratory conditions, we choice following typical parameters,

$$
\begin{aligned}
& \hat{B}_{y}^{(0)}=10 \mathrm{~T}, \mathrm{~L}=10 \mathrm{~m}, \\
& v_{e}=v_{g}=5 \mathrm{GHz}\left(\lambda_{g}=0.06 m, k_{e}=k_{g}=\frac{2 \pi}{\lambda} \approx 100\right), \\
& h v=3.3 \times 10^{-24} J(\text { energy of single photon), } \\
& A \approx h_{r m s}=\hat{h}=10^{-26} \text { to } 10^{-30}, \\
& \Delta s=0.1 \times 0.1=0.01 \mathrm{~m}^{2} \text { (typical receiving surface), }
\end{aligned}
$$

where $\Delta s$ is also the cross section of the interacting region. If $\hat{h}=h_{r m s}=$ $10^{-30}$, then the total power flux passing through $\Delta s$ in the terminal position $(\mathrm{z}=\mathrm{L})$ is

$$
U_{e m}^{(2)}=u_{e m} \Delta s=\frac{1}{\mu_{0}}\left(A \hat{B}_{y}^{(0)} k_{g} L\right)^{2} c \Delta s \approx 2.3 \times 10^{-40} \mathrm{~W},
$$

where the superscript (2) denotes the second-order perturbative EM power flux. Therefore, corresponding second-order perturbative photon flux (in quantum language) will be

$$
N_{\gamma}^{(2)}=U_{e m}^{(2)} / \hbar \omega_{e} \approx 2.3 \times 10^{-40} / 3.3 \times 10^{-24} \approx 7.0 \times 10^{-17} s^{-1} .
$$

For an HFGW of $v_{g}=5 \mathrm{GHz}, \hat{h}=10^{-30}$, the total power flux passing through the $\Delta s$ is given by [36]

$$
U_{g w}=u_{g w} \Delta s=\frac{c^{3}}{8 \pi G} \omega^{2} A^{2} \Delta s \approx 1.6 \times 10^{-7} \mathrm{~W},
$$

Thus corresponding graviton flux would be

$$
N_{g}=U_{g w} / \hbar \omega \approx 4.8 \times 10^{16} s^{-1} .
$$

Because the power fluxes, Eq.(7) (including the photon flux, Eq.(8)) is proportional to the amplitude squared of the HFGW, the second-order perturbative photon flux (PPF) exhibits a very small value.

From Eqs.(7)-(10), we obtain the conversion rate of the HFGW (gravitons) into the EMW (photons) as follows

$$
P \approx U_{e m} / U_{g w}=N_{\gamma} / N_{g}=\frac{2.3 \times 10^{-40}}{1.6 \times 10^{-7}}=\frac{7 \times 10^{-17}}{4.8 \times 10^{16}} \approx 1.4 \times 10^{-33} .
$$


Eqs.(2) and (11) show that the conversion rates of the EMW (photons) into the HFGW (gravitons) and the contrary process have similar orders of magnitude. Thus, in order to obtain a second-order perturbative photon, from Eq. (8), the signal accumulation time would be, at least

$$
\Delta t \approx 1 / N_{r}^{(2)} \approx \frac{1}{7 \times 10^{-17}} \approx 1.4 \times 10^{16} \mathrm{~s} .
$$

This is a very huge time interval. Eqs.(11) and (12) also show that the conversion rate of the HFGW (gravitons) into the EMW (photons) is extremely low. Thus the PPF in the pure inverse G-effect cannot cause a detectable signal or observable effect in the laboratory condition. Nevertheless, for some astrophysical and cosmological processes, it is possible to cause interesting phenomena, because the very large EM fields (including plasma) and very strong GWs (including low frequency GWs) often occur simultaneously and these fields extend over a very large area $[15,37,38]$.

From Eqs. (5) (7), (8) and (12), one finds,

$$
\begin{aligned}
& \text { if } \hat{h}=10^{-26} \text {, then } N_{\gamma}^{(2)} \approx 7 \times 10^{-9} \mathrm{~s}^{-1} \text { and } \Delta t \approx 1.4 \times 10^{8} \mathrm{~s} \text {, } \\
& \hat{h}=10^{-24}, \text { then } N_{\gamma}^{(2)} \approx 7 \times 10^{-5} \mathrm{~s}^{-1} \text { and } \Delta t \approx 1.4 \times 10^{4} \mathrm{~s} \text {. }
\end{aligned}
$$

Such results show that even if $\hat{h}=10^{-24}$, it is still difficult to detect the HFGWs by the inverse G-effect in the laboratory condition. In other words, in order to generate an observable effect in such EM system, the amplitude of the HFGW of $v_{g}=5 \mathrm{GHz}$ must be larger than $\hat{h}=10^{-24}$ at least. Unfortunately, so far as, we know there are no those HFGWs as strong as $\hat{h}=10^{-24}$ or larger, though the EM system based on the pure inverse G-effect in the high-vacuum and ultra-low-temperature condition has a very good low noise environment. Therefore the EM detecting scheme based the pure inverse G-effect in the laboratory condition would not be available to detect HFGWs in the microwave band.

\section{The perturbative photon fluxes in coupling system be- tween the static magnetic field and the plane EMW.}

The classical and semi-classical description and linear quantum theory all showed $[33,39]$ that the interaction cross section between the $\mathrm{GW}$ (gravitons) and the EMW(photons) in a strong background static mag- 
netic field (virtual photons) will be much larger than that in the pure inverse G-effect. In other words, the strong background static magnetic field provides a catalyst to greatly enhance the resonant effect between the EMW (the photons) and the GW (gravitons). However, the presence of background EMW (the background photon flux) will generate a large photon flux noise. If the perturbative photon flux (PPF, i.e., signal photon flux) and the background photon flux (BPF) have the same or the very similar physical behaviors (e.g., propagating direction, distribution, decay rate, etc.), then the PPF will be swamped by the BPF. The coupling system between a plane EMW and the static magnetic field is just this case (see Fig.2), which will have the same or very similar sensitivity as the inverse G-effect. We assume the power of the background EMW is $10 \mathrm{~W}$, and it is limited in the cross section of $\Delta s=0.1 \times 0.1=0.01 \mathrm{~m}^{2}$. Because the power flux of the plane EMW is distributed homogeneously in the cross section $\Delta s$, then

$$
\begin{aligned}
& \left\langle P_{e m}\right\rangle=\operatorname{Re}\left(\frac{1}{2 \mu_{0}} E_{x}^{*(0)} B_{y}^{(0)}\right) \Delta s=\frac{1}{2 \mu_{0}} \frac{E_{x}^{(0)^{2}}}{c} \Delta s=10 \mathrm{~W}, \\
& \text { and } \quad\left|\vec{E}_{x}^{(0)}\right| \approx 8.7 \times 10^{2} \mathrm{Vm}^{-1} .
\end{aligned}
$$

Total background photon flux passing through the cross section $\Delta s$ will be

$$
N_{\gamma}^{(0)}=10 / \hbar \omega_{e}=\frac{10}{3.3 \times 10^{-24}} \approx 3.0 \times 10^{24} \mathrm{~s}^{-1} \text {. }
$$

Then corresponding first-order perturbative power flux in the z-direction is

$$
\begin{aligned}
U_{z}^{(1)}= & \frac{1}{2 \mu_{0}}\left[\left(\vec{E}^{(1)} \times \vec{B}_{y}^{(0)}\right)+\left(\vec{E}_{x}^{(0)} \times \vec{B}^{(1)}\right)\right]_{\omega_{e}=\omega_{g}} \Delta s . \\
& =\operatorname{Re}\left[\frac{1}{\mu_{0}} E^{(1) *} B_{y}^{(0)}\right] \cos \beta \cos \delta \cdot \Delta s \\
& =\operatorname{Re}\left[\frac{1}{\mu_{0} c} E^{(1) *} E_{x}^{(0)}\right] \cos \beta \cos \delta \cdot \Delta s \\
& =\frac{1}{\mu_{0} c}\left|\vec{E}^{(1)} \| \vec{E}_{x}^{(0)}\right| \cos \beta \cos \delta \cdot \Delta s,
\end{aligned}
$$

where $\delta$ is the phase difference between the HFGW and the background $\mathrm{EMW}_{0}, \beta$ is the angle between $\vec{E}^{(1)}$ and $\vec{E}_{x}^{(0)}$ or $\vec{B}^{(1)}$ and $\vec{B}_{y}^{(0)}$ (see Fig.3). Here $\delta=0$ and $\beta=0$ will always be possible by regulating the phase and the polarization directions of the background $\mathrm{EMW}_{0}$. Then the HFGW and the EMW will have the best matching state, i.e., 

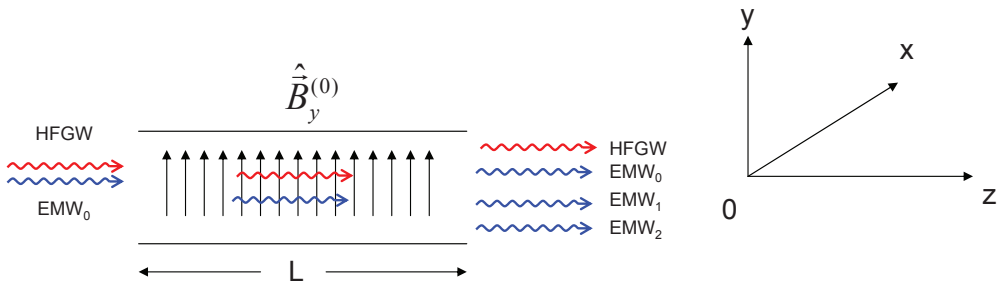

Figure 2: If the HFGW and the $E M W_{0}$ pass simultaneously through the transverse static magnetic field, under the resonant state $\left(\omega_{e}=\omega_{g}\right)$, the first-order perturbative EMW ( $E M W_{1}$, i.e., "the interference term") and the second-order perturbative EMW (the $E M W_{2}$ ) can be generated. However, because the $E M W_{1}$ and the $E M W_{0}$ have the same propagating direction and distribution, and $E M W_{1}$ is often much less than the $E M W_{0}$, the $E M W_{1}$ will be swamped by the $E M W_{0}$ 


$$
\left.U_{z}^{(1)}\right|_{\substack{\delta=0 \\ z=L}}=U_{z \max }^{(1)}=\operatorname{Re}\left[\frac{1}{\mu_{0} c} E^{(1) *} E_{x}^{(0)}\right] \Delta s \approx 6.9 \times 10^{-20} \mathrm{~W} .
$$

Then the corresponding first-order PPF will be

$$
N_{z}^{(1)}=U_{z}^{(1)} / \hbar \omega_{e} \approx 6.9 \times 10^{-20} / 3.3 \times 10^{-24} \approx 2.1 \times 10^{4} \mathrm{~s}^{-1} \text {. }
$$

Thus the total photon flux passing through $\Delta s$ is about

$$
N_{z}=N_{z}^{(0)}+N_{z}^{(1)}+N_{z}^{(2)} \approx\left(3.0 \times 10^{24}+2.1 \times 10^{4}+7.0 \times 10^{-17}\right) \mathrm{s}^{-1} .
$$

In this case the ratio of $N_{z}^{(1)}$ and $N_{z}^{(0)}$ is roughly

$$
\sigma_{1}=N_{z}^{(1)} / N_{z}^{(0)} \approx \frac{2.1 \times 10^{4}}{3.0 \times 10^{24}} \approx 7.0 \times 10^{-21}
$$

This is also very small value, and at the same time,

$$
\sigma_{2}=N_{z}^{(2)} / N_{z}^{(1)} \approx \frac{7.0 \times 10^{-17}}{2.1 \times 10^{4}} \approx 3.3 \times 10^{-21}
$$

i.e., the second-order PPF is much less than the first-order PPF, while the first-order PPF is much less than the background photon flux (BPF). This means that if an EM detecting system contains simultaneously the static magnetic field and the EMW, then the interaction cross section between the GW (gravitons) and the EMW (photons) will be much larger than that in the pure inverse G-effect. The classical description and linear quantum theory for such a property have good self-consistency [33,39].

However, Eqs. (3),(4),(14),(16),(18) show that the first-order PPF (signal) and the BPF (noise) have the same propagating direction and distribution, and the BPF is much larger than the PPF, so that the PPF will be swamped by the BPF. In this case the PPF has no direct observable effect. According to Eqs. (3), (4), (17) and (18), one finds

$$
\begin{aligned}
& \text { if } \hat{h}=10^{-26}, \text { then } N_{z}^{(1)} \approx 2.1 \times 10^{8} \mathrm{~s}^{-1}, \\
& \text { if } \hat{h}=10^{-25} \text {, then } N_{z}^{(1)} \approx 2.1 \times 10^{9} \mathrm{~s}^{-1} .
\end{aligned}
$$

For example, if $\hat{h}=10^{-26}$, in order to displaying first-order PPF, $N_{z}^{(1)} \Delta t$ must be effectively larger than the background noise fluctuation $\sqrt{N_{z}^{(0)} \Delta t}$, i.e., 


$$
\begin{aligned}
& N_{z}^{(1)}(\Delta t)^{\frac{1}{2}}>\sqrt{N_{z}^{(0)}}, \\
& \text { then } \Delta t>6.8 \times 10^{7} \mathrm{~s},
\end{aligned}
$$

where $N_{z}^{(0)} \Delta t$ is the expectation value with a Poisson distribution of width $\sqrt{N_{z}^{(0)}}$. Eqs. (13) and (23) show that such two schemes have similar detecting sensitivity. Thus, detecting the HFGW of $\hat{h}=10^{-26}$ and $v=5 \mathrm{GHz}$ by such coupling EM system will also be very difficult.

IV. Coupling system of the static magnetic field and the Gaussian type microwave photon flux

The above discussion shows that in order to detect the first-order PPF, one must find a special EM resonant system in which the PPF and the BPF have very different physical behaviors, even if such difference are only distributed in a few local regions.

Before we discuss the resonance effect of the HFGWs in the proposal EM system, we give a general analysis of the photon flux. Here, $\vec{E}^{(0)}, \vec{B}^{(0)}$ denote the background EM fields, $\vec{E}^{(1)}, \vec{B}^{(1)}$ the perturbative EM fields produced by the interaction of the HFGW with the static magnetic field. Then total EM power flux density is

$$
\begin{aligned}
& \vec{u}_{e m}=\frac{1}{\mu_{0}} \vec{E} \times \vec{B}=\frac{1}{\mu_{0}}\left(\vec{E}^{(0)}+\vec{E}^{(1)}\right) \times\left(\vec{B}^{(0)}+\vec{B}^{(1)}\right) \\
& =\frac{1}{\mu_{0}} \vec{E}^{(0)} \times \vec{B}^{(0)}+\frac{1}{\mu_{0}}\left(\vec{E}^{(0)} \times \vec{B}^{(1)}+\vec{E}^{(1)} \times \vec{B}^{(0)}\right)+\frac{1}{\mu_{0}} \vec{E}^{(1)} \times \vec{B}^{(1)} .
\end{aligned}
$$

Thus, the corresponding total photon flux density will be

$$
\begin{aligned}
& \vec{n}_{\gamma}=\frac{1}{\hbar \omega_{e}} \vec{u}_{e m} \\
& =\frac{1}{\mu_{0} \hbar \omega_{e}}\left(\vec{E}^{(0)} \times \vec{B}^{(0)}\right)+\frac{1}{\mu_{0} \hbar \omega_{e}}\left(\vec{E}^{(0)} \times \vec{B}^{(1)}+\vec{E}^{(1)} \times \vec{B}^{(0)}\right) \\
& +\frac{1}{\mu_{0} \hbar \omega_{e}}\left(\vec{E}^{(1)} \times \vec{B}^{(1)}\right) \\
& =\vec{n}^{(0)}+\vec{n}^{(1)}+\vec{n}^{(2)}
\end{aligned}
$$

where

$$
\begin{aligned}
& \vec{n}^{(0)}=\frac{1}{\mu_{0} \hbar \omega_{e}}\left(\vec{E}^{(0)} \times \vec{B}^{(0)}\right), \\
& \vec{n}^{(1)}=\frac{1}{\mu_{0} \hbar \omega_{e}}\left(\vec{E}^{(0)} \times \vec{B}^{(1)}+\vec{E}^{(1)} \times \vec{B}^{(0)}\right), \\
& \vec{n}^{(2)}=\frac{1}{\mu_{0} \hbar \omega_{e}}\left(\vec{E}^{(1)} \times \vec{B}^{(1)}\right) .
\end{aligned}
$$


Eq. (25) and (26) would be most general form of the PPF and the BPF, where $\vec{n}^{(0)}, \vec{n}^{(1)}$ and $\vec{n}^{(2)}$ express the BPF, the first-order PPF and the secondorder PPF densities, respectively. Since non-vanishing $\left|\vec{E}^{(0)}\right|,\left|\vec{B}^{(0)}\right|$ are often much larger than $\left|\vec{E}^{(1)}\right|,\left|\vec{B}^{(1)}\right|$, we have

$$
\left|\vec{n}^{(0)}\right| \gg\left|\vec{n}^{(1)}\right| \gg\left|\vec{n}^{(2)}\right| \text {. }
$$

4-1.In the case of the plane Electromagnetic Wave or the plane EMW.

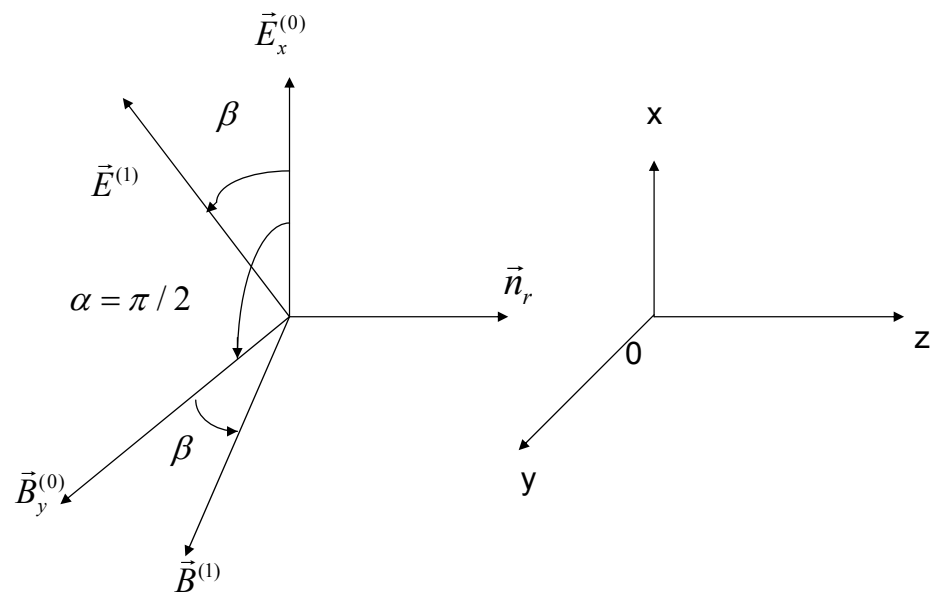

Figure 3: In the coupling system of the static magnetic field and the plane EMW, $\left|\vec{E}_{x}^{(0)}\right|$ and $\left|\vec{B}_{y}^{(0)}\right|$ denote the background EM fields, $\left|\vec{E}^{(1)}\right|$ and $\left|\vec{B}^{(1)}\right|$ express the perturbative EM fields generated by the direct interaction of the HFGW with the static magnetic field, $\vec{n}_{\gamma}$ is the total photon flux density.

If the $\mathrm{HFGW}$ and the plane $\mathrm{EMW}_{0}$ all propagate along the z-direction, then Eq.(25) is deduced to (see Fig.3) 


$$
\begin{aligned}
n_{\gamma} & =\left\langle\vec{n}_{\gamma}\right\rangle_{\omega_{e}=\omega_{g}}=\frac{1}{2 \mu_{0} \hbar \omega_{e}}\left\langle\left(\vec{E}_{x}^{(0)}+\vec{E}^{(1)}\right) \times\left(\vec{B}_{y}^{(0)}+\vec{B}^{(1)}\right)\right\rangle_{\omega_{e}=\omega_{g}} \\
& =\frac{1}{2 \mu_{0} \hbar \omega_{e}}\left\{\left|\vec{E}_{x}^{(0)}\right|\left|\vec{B}_{y}^{(0)}\right|+\left[\left|\vec{E}_{x}^{(0)} \| \vec{B}^{(1)}\right| \sin \left(\frac{\pi}{2}+\beta\right)\right.\right. \\
& \left.\left.+\left|\vec{E}^{(1)} \| \vec{B}_{y}^{(0)}\right| \sin \left(\frac{\pi}{2}-\beta\right)\right] \cos \delta+\left|\vec{E}^{(1)} \| \vec{B}^{(1)}\right|\right\} .
\end{aligned}
$$

where the angular bracket denotes the average over time. For the plane EMW in empty space, $B_{y}^{(0)}=E_{x}^{(0)} / c, B^{(1)}=E^{(1)} / c$ (in MKS units), then Eq.(28) becomes

$$
\begin{aligned}
n_{\gamma} & =\frac{1}{2 \mu_{0} c \hbar \omega_{e}}\left\{\left|\vec{E}_{x}^{(0)}\right|^{2}+2\left|\vec{E}_{x}^{(0)}\right|\left|\vec{E}^{(1)}\right| \cos \beta \cos \delta+\left|\vec{E}^{(1)}\right|^{2}\right\} \\
& =\frac{1}{2 \mu_{0} c \hbar \omega_{e}}\left\{\left|\vec{E}_{x}^{(0)}\right|^{2}+2 \vec{E}_{x}^{(0)} \cdot \vec{E}^{(1)} \cos \delta+\left|\vec{E}^{(1)}\right|^{2}\right\} \\
& =n^{(0)}+n^{(1)}+n^{(2)},
\end{aligned}
$$

where

$$
\begin{aligned}
& n^{(0)}=\frac{1}{2 \mu_{0} c \hbar \omega_{e}}\left|\vec{E}_{x}^{(0)}\right|^{2}, \\
& n^{(1)}=\frac{1}{\mu_{0} c \hbar \omega_{e}} \vec{E}_{x}^{(0)} \cdot \vec{E}^{(1)} \cos \delta, \\
& n^{(2)}=\frac{1}{2 \mu_{0} c \hbar \omega_{e}}\left|\vec{E}^{(1)}\right|^{2} .
\end{aligned}
$$

In fact, Eq.(30) can also be expressed as

$$
\begin{aligned}
& n^{(0)}=\frac{1}{2 \mu_{0} c \hbar \omega_{e}}\left|\vec{E}_{x}^{(0)}\right|^{2}=\dot{N}_{0} \text { (the background photon flux density) } \\
& n^{(2)}=\frac{1}{2 \mu_{0} c \hbar \omega_{e}}\left|\vec{E}^{(1)}\right|^{2}=\dot{N}_{G W}(\text { the second }- \text { order PPF density) }
\end{aligned}
$$

while

$$
n^{(1)}=\frac{1}{\mu_{0} c \hbar \omega_{e}} \vec{E}_{x}^{(0)} \cdot \vec{E}^{(1)} \cos \delta=2\left(\dot{N}_{0} \dot{N}_{G W}\right)^{\frac{1}{2}} \cos \delta=\dot{N}_{1}
$$

(the interference term, i.e., the first - order PPF density).

Then, Eq.(29) can be re-written as

$$
n_{\gamma}=\dot{N}_{0}+2\left(\dot{N}_{0} \dot{N}_{G W}\right)^{\frac{1}{2}} \cos \delta+\dot{N}_{G W}
$$

After a long time interval $\Delta t$ the collected number of photons at the detector or at the receiving surface would be 


$$
N_{d}=n_{\gamma} \Delta t=\dot{N}_{0} \Delta t+2\left(\dot{N}_{0} \dot{N}_{G W}\right)^{\frac{1}{2}} \cos \delta \cdot \Delta t+\dot{N}_{G W} \Delta t
$$

Clearly, in the plane EMW case, the BPF, the first-order PPF and the second-order PPF all propagate along the same direction, thus in any region and at any receiving surface

$$
\dot{N}_{0} \gg 2\left(\dot{N}_{0} \dot{N}_{G W}\right)^{\frac{1}{2}} \gg \dot{N}_{G W}
$$

is always valid. In this case, it is very difficult to display the first-order PPF effect $\left(n^{(1)}=2\left(\dot{N}_{0} \dot{N}_{G W}\right)^{\frac{1}{2}} \cos \delta=\dot{N}_{1}\right)$ in an acceptable signal accumulation time interval with the predicted total photon flux background.

In the coupling system between the Gaussian type-microwave photon flux (Gaussian beam (GB) is just one typical form of the Gaussian type-microwave photon fluxes) and the static magnetic field, the general expressions, Eqs.(25) and (26) are still valid. However, they will be expressed as the different exact forms in the different directions and the receiving surfaces, and the relative relation between $n^{(0)}$ and $n^{(1)}$ would be different in the different receiving surfaces, even then they can reach up a comparable order of magnitude. This is worth consideration. The scheme from [32] would be a useful candidate(see Fig. 4). Thus key parameters in the scheme are the BPF and the first-order PPF in the special directions and not the photon number. The former are vectors and have high directivity. They decide the strength of the photon fluxes reaching the detector or the receiving surface, position and bearings of the detectors and the signal-to-noise ratio (SNR) in the receiving surfaces.

4.2 Coupling system of the Gaussian-type microwave photon flux and the static magnetic field.

Unlike plane EMW, the GB has not only longitudinal BPF (the BPF in the z-direction, i.e., the direction of its symmetrical axis) but also the transverse BPF, although the latter is often less than the former. The $\mathrm{BPF}$ in the transverse directions (e.g., the $\mathrm{x}$ - and $\mathrm{y}$ - direction) decays as fast as the typical Gaussian decay rate. Thus in the some special regions and directions, the effect of both the PPF and the BPF would have a comparable order of magnitude.

For the GB with the double transverse polarized electric modes[32,40] it has 


$$
\begin{aligned}
& \vec{E}^{(0)}=\vec{E}_{x}^{(0)}+\vec{E}_{y}^{(0)}, \\
& \vec{B}^{(0)}=\vec{B}_{x}^{(0)}+\vec{B}_{y}^{(0)}+\vec{B}_{z}^{(0)} .
\end{aligned}
$$

Such EM fields satisfy the Helmholtz equation. If the circular polarized HFGW propagates along the z-direction, then the non-vanishing perturbative EM fields are $\vec{E}_{x}^{(1)}, \vec{B}_{y}^{(1)}$ (the perturbative EM fields produced by the $\oplus$ polarization component of the HFGW) and $\vec{E}_{y}^{(1)}, \vec{B}_{x}^{(1)}$ (the perturbative EM fields generated by the $\otimes$ polarization component of the HFGW) in our scheme[32], respectively, i.e.,

$$
\begin{aligned}
& \vec{E}^{(1)}=\vec{E}_{x}^{(1)}+\vec{E}_{y}^{(1)}, \\
& \vec{B}^{(1)}=\vec{B}_{x}^{(1)}+\vec{B}_{y}^{(1)} .
\end{aligned}
$$

In this case, Eq.(25) has following concrete expression

$$
\begin{aligned}
& \vec{n}_{\gamma}=\frac{1}{\mu_{0} \hbar \omega_{e}} \vec{E} \times \vec{B} \\
& =\frac{1}{\mu_{0} \hbar \omega_{e}}\left\{\left(\vec{E}_{x}^{(0)}+\vec{E}_{x}^{(1)}+\vec{E}_{y}^{(0)}+\vec{E}_{y}^{(1)}\right) \times\left(\vec{B}_{x}^{(0)}+\vec{B}_{x}^{(1)}+\vec{B}_{y}^{(0)}+\vec{B}_{y}^{(1)}+\vec{B}_{z}^{(0)}\right)\right\} .
\end{aligned}
$$

From Eq. (38), under the resonant state $\left(\omega_{e}=\omega_{g}\right)$ the total photon flux densities in the $\mathrm{z}$-direction (the longitudinal direction of the GB) and in the transverse direction (the $\mathrm{x}$ - and $\mathrm{y}$ - directions) can be given by

$$
\begin{aligned}
n_{z} & =\frac{1}{2 \mu_{0} \hbar \omega_{e}} \operatorname{Re}\left\{\left[E_{x}^{*(0)} B_{y}^{(0)}+E_{y}^{*(0)} B_{x}^{(0)}\right]\right. \\
& +\left[E_{x}^{*(0)} B_{y}^{(1)}+E_{y}^{*(0)} B_{x}^{(1)}+E_{x}^{*(1)} B_{y}^{(0)}+E_{y}^{*(1)} B_{x}^{(0)}\right] \\
& \left.+\left[E_{x}^{*(1)} B_{y}^{(1)}+E_{y}^{*(1)} B_{x}^{(1)}\right]\right\} \\
& =n_{z}^{(0)}+n_{z}^{(1)}+n_{z}^{(2)} \\
& =n_{z}^{(0)}+n_{z}^{(1)}+o\left(h^{2}\right), \\
n_{x} & =\frac{1}{2 \mu_{0} \hbar \omega_{e}} \operatorname{Re}\left[E_{y}^{*(0)} B_{z}^{(0)}+E_{y}^{*(1)} B_{z}^{(0)}\right]=n_{x}^{(0)}+n_{x}^{(1)}, \\
n_{y} & =\frac{1}{2 \mu_{0} \hbar \omega_{e}} \operatorname{Re}\left[E_{x}^{*(0)} B_{z}^{(0)}+E_{x}^{*(1)} B_{z}^{(0)}\right]=n_{y}^{(0)}+n_{y}^{(1)} .
\end{aligned}
$$


(1)The photon flux in the z-direction (the longitudinal direction of the GB)

From Eq. (39) and Refs.[32,40], we have

$$
n_{z}^{(0)}=\left|n_{z}^{(0)}\right|_{\max } \exp \left(-\frac{2 r^{2}}{W^{2}}\right), \quad n_{z}^{(1)}=\left|n_{z}^{(1)}\right|_{\max } \exp \left(-\frac{r^{2}}{W^{2}}\right),
$$

where $\mathrm{r}$ is the radial distance to the symmetrical axis (the $\mathrm{z}$-axis) of the $\mathrm{GB}, W$ is the spot radius of the GB. Eq. (42) shows that $n_{z}^{(0)}$ decays by the typical Gaussian decay rate $\exp \left(-\frac{2 r^{2}}{W^{2}}\right)$, while $n_{z}^{(1)}$ decays by the factor $\exp \left(-\frac{r^{2}}{W^{2}}\right)$, i.e., the decay rate of $n_{z}^{(1)}$ is slower than that of $n_{z}^{(0)}$. However, since $\left|n_{z}^{(0)}\right|_{\max } \gg\left|n_{z}^{(1)}\right|_{\max }$ in almost all of the regions (see Fig.5), it is difficult to generate an observable effect by $n_{z}^{(1)}$ in these regions. For the HFGW parameters of $h=10^{-30}, v=5 \mathrm{GHz}$, only if $r \rightarrow 34 \mathrm{~cm}$ (at the xy plane), $n_{z}^{(1)}$ has comparable order of magnitude with $n_{z}^{(0)}$. However, $n_{z}^{(1)}$ and $n_{z}^{(0)}$ all are decayed to the very small undetectable value $n_{z}^{(1)} \sim n_{z}^{(0)} \sim$ $10^{-16} \mathrm{~s}^{-1} \mathrm{~m}^{-2}$. 


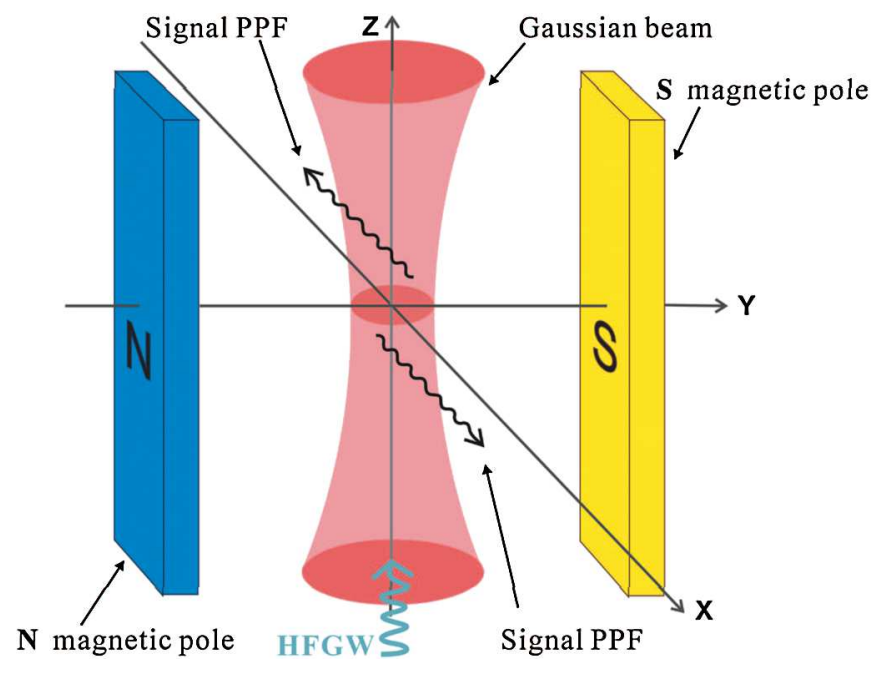

Figure 4: When the HFGW propagates along the z-direction in the coupling system of the GB and the transverse static magnetic field $\hat{\vec{B}}_{y}(0)$, the resonant interaction $\left(\omega_{e}=\omega_{g}\right)$ of the HFGW with the EM fields will generate not only the longitudinal perturbative photon flux $n_{z}^{(1)}$, but also the transverse perturbative photon fluxes $\left(n_{x}^{(1)}\right.$ and $\left.n_{y}^{(1)}\right)$ in the x- and y-directions due to the spread property of the GB itself. This is an important difference between Fig.2 and Fig.4. Moreover, unlike $n_{z}^{(1)}$ and $n_{z}^{(0)}, n_{x}^{(1)}$ and $n_{x}^{(0)}$ have very different distribution and the decay rates. 


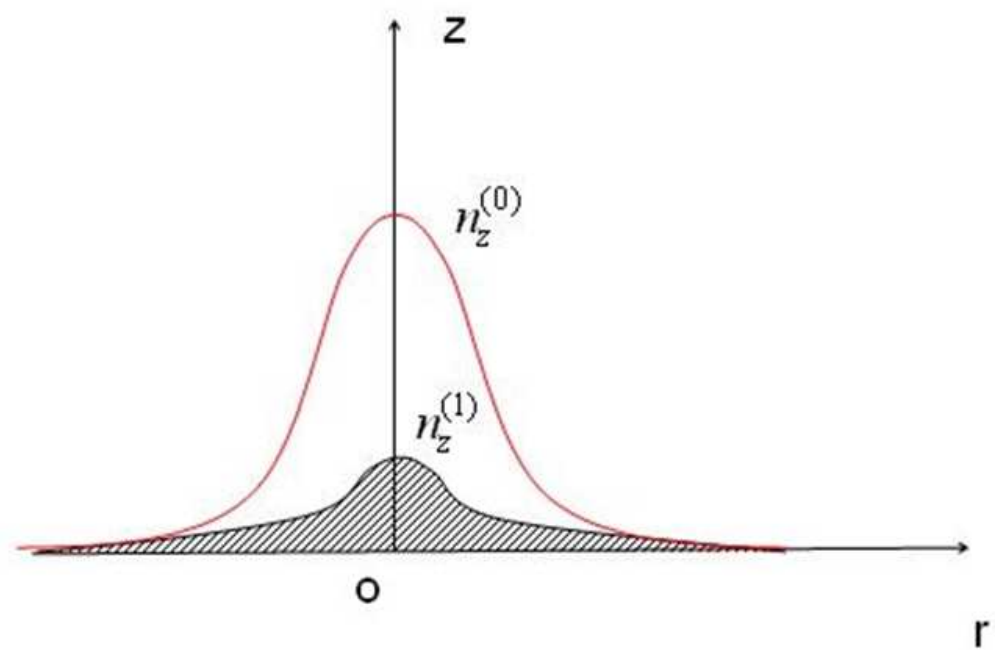

Figure 5: The first-order PPF density $n_{z}^{(1)}$ and the BPF density $n_{z}^{(0)}$ have the same propagating direction and the similar distribution. Thus $n_{z}^{(0)}$ is much larger than $n_{z}^{(1)}$ in most of the regions.

(2)The photon fluxes in the $\mathrm{x}$-direction (the transverse direction of the GB).

According to Eq. (40) and Refs.[32,40], one finds

$$
n_{x}=\frac{1}{2 \mu_{0} \hbar \omega_{e}}\left\langle\left|\vec{E}_{y}^{(0)}\left\|\vec{B}_{z}^{(0)}|+| \vec{E}_{y}^{(1)}\right\| \vec{B}_{z}^{(0)}\right| \cos \delta\right\rangle_{\omega_{e}=\omega_{g}},
$$

Setting $\delta=0$ will always be possible by regulating the phase of the GB. Then

$$
\begin{aligned}
& n_{x}=\frac{1}{2 \mu_{0} \hbar \omega_{e}}\left\{\left\langle\vec{E}_{y}^{(0)} \vec{B}_{z}^{(0)}\right\rangle+\left\langle\vec{E}_{y}^{(1)} \vec{B}_{z}^{(0)}\right\rangle\right\}_{\omega_{e}=\omega_{g}} \\
& =n_{x}^{(0)}+n_{x}^{(1)}=\dot{N}_{0 x}+\dot{N}_{1 x}
\end{aligned}
$$

where

$$
\begin{gathered}
n_{x}^{(0)}=\dot{N}_{0 x}=\frac{1}{2 \mu_{0} \hbar \omega_{e}}\left\langle\left|\vec{E}_{y}^{(0)}\right| \mid \vec{B}_{z}^{(0) \mid}\right\rangle=\left|n_{x}^{(0)}\right|_{\max } x \exp \left(-\frac{2 x^{2}}{W^{2}}\right), \\
n_{x}^{(1)}=\dot{N}_{1 x}=\frac{1}{2 \mu_{0} \hbar \omega_{e}}\left\langle\left|\vec{E}_{y}^{(1)}\right|\left|\vec{B}_{z}^{(0)}\right|\right\rangle_{\omega_{e}=\omega_{g}}=\left|n_{x}^{(1)}\right|_{\max } \exp \left(-\frac{x^{2}}{W^{2}}\right),
\end{gathered}
$$


Unlike the case of plane EMW, Eqs. (45) and (46) show that $\dot{N}_{0 x}$ will be not always larger than $\dot{N}_{1 x}$. In the case of GB, $B_{z}^{(0)}$ of the GB depends not only on $\vec{E}_{y}^{(0)}$, but also $\vec{E}_{x}^{(0)}$, i.e.,

$$
B_{z}^{(0)}=\frac{i}{\omega_{e}}\left(\frac{\partial E_{x}^{(0)}}{\partial y}-\frac{\partial E_{y}^{(0)}}{\partial x}\right) .
$$

Therefore, when $E_{y}^{(0)}=0, n_{x}^{(0)}$ must be vanish, but $n_{x}^{(1)}=n_{x \max }^{(1)} \neq 0$

Although Eqs. (45) and (46) all represent the transverse photon fluxes in the $\mathrm{x}$-direction, but their physical behaviors are quite different:

1. At the yz-plane $\left.n_{x}^{(1)}\right|_{x=0}=\left.n_{x}^{(1)}\right|_{\max }$ where $\left.n_{x}^{(0)}\right|_{x=0}=0$, i.e., the transverse PPF has a maximum at the longitudinal symmetrical surface of the GB where the transverse BPF vanishes. It should be pointed out that, the transverse BPF at the longitudinal symmetrical surfaces being identically to zero is a fundamental characteristics of the GB's, whether the circular or elliptic GB's. Thus the transverse PPF would be a major fraction of the total transverse photon fluxes flux passing through such a surface, provided the other noise photon flux passing through the surface can be effectively suppressed, although the PPF is much less than the BPF in other regions, and the PPF is always accompanied simultaneously by the BPF.

2. The $n_{x}^{(1)}$ and $n_{x}^{(0)}$ have different decay rates in the $\mathrm{x}$-direction, i.e., $n_{x}^{(1)} \propto \exp \left(-\frac{x^{2}}{\mathrm{~W}^{2}}\right), n_{x}^{(0)} \propto x \exp \left(-\frac{2 x^{2}}{\mathrm{~W}^{2}}\right)$. The position of a maximum of $n_{x}^{(1)}$ is the yz plane $(\mathrm{x}=0)$, while the position of maximum of $n_{x}^{(0)}$ is about $\mathrm{x}=3.2 \mathrm{~cm}$ in our case. Thus, SNR $n_{x}^{(1)} / n_{x}^{(0)}$ will be very different at the different receiving surfaces. This means that it is always possible to obtain a best SNR $n_{x}^{(1)} / n_{x}^{(0)}$ by choosing the suitable region and the receiving surface. Using Eqs.(45) and (46), the total transverse photon fluxes passing through the receiving surface $\Delta s$ can be given by

$$
\begin{aligned}
& N_{x}^{(1)}=\int_{\Delta s} n_{x}^{(1)} d s, \\
& N_{x}^{(0)}=\int_{\Delta s} n_{x}^{(0)} d s,
\end{aligned}
$$

In the current scheme, $\Delta s \approx 10^{-2} \mathrm{~m}^{2}$. 


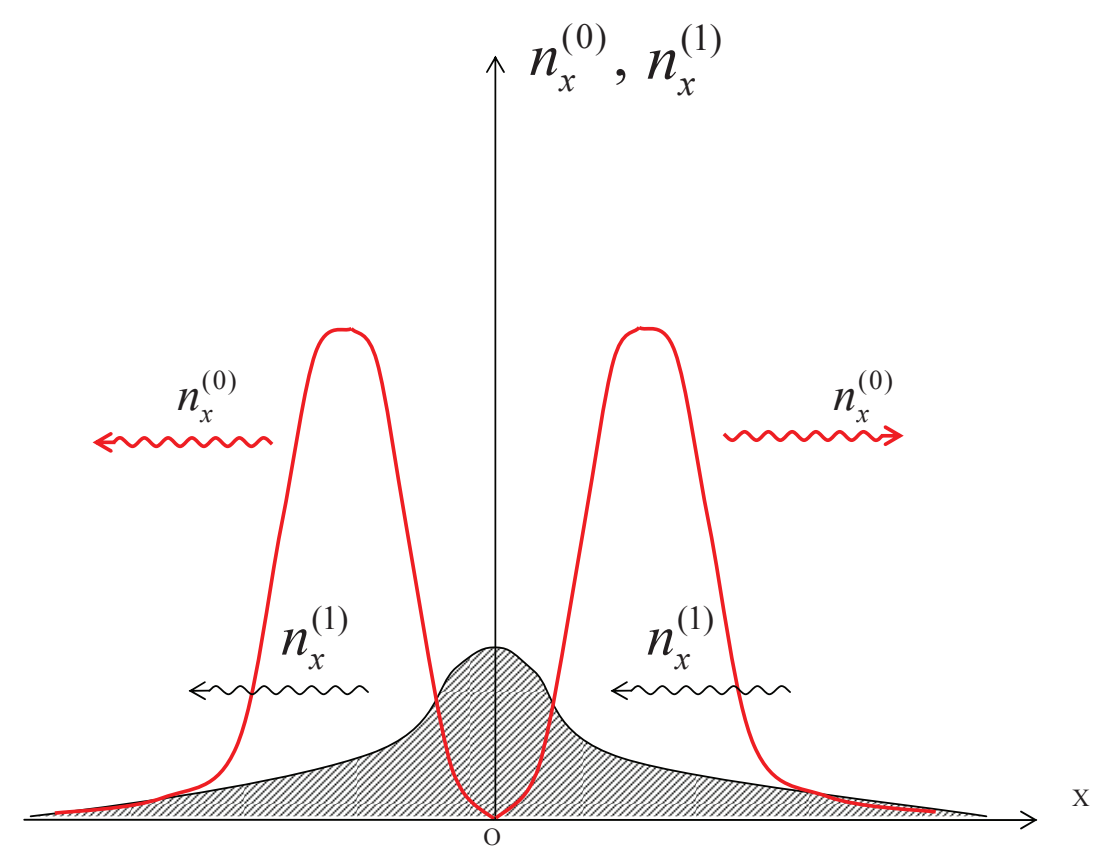

Figure 6: Schematic diagram of strength distribution of $n_{x}^{(0)}$ and $n_{x}^{(1)}$ in the "outgoing wave" region of the GB (another one is the "imploding wave" region. For an optimum GB, such properties of the transverse BPFs in such two regions would be "anti-symmetric"). Unlike Fig.5, here $\left.n_{x}^{(0)}\right|_{x=0}=0$ while $\left.n_{x}^{(1)}\right|_{x=0}=\left.n_{x}^{(1)}\right|_{\max }$. Therefore, $n_{x}^{(1)} \Delta t$ can be effectively larger than the background noise photon flux fluctuation $\left(n_{x}^{(0)} \Delta t\right)^{1 / 2}$, i.e., $n_{x}^{(1)} \Delta t>\left(n_{x}^{(0)} \Delta t\right)^{1 / 2}$ at the yz-plane and at the parallel surfaces near the yz-plane, and $n_{x}^{(1)}$ will be major fraction of the total transverse photon flux passing through the yz-plane, provided thermal photon flux and other noise photon fluxes passing through the surface can be effectively suppressed. Clearly, the EM response of the coupling system between the plane EMW and the static magnetic field has no such characteristic. Moreover, the propagating directions of $n_{x}^{(1)}$ are opposite in the regions of $y>0$ and $y<0$ for our scheme. Thus, the total momentum of the PPF in the $\mathrm{x}$-direction vanishes. In other words, such a property ensured conservation of the total momentum in the coherent resonance interaction (see Ref.[32]). 
4-3 Numerical estimation of the transverse photon fluxes.

In order to measure $N_{x}^{(1)}$ at a suitable receiving surface, $N_{x}^{(1)} \Delta t$ (notice that here $N_{x}^{(1)}$ is equivalent to $2\left(\dot{N}_{0} \dot{N}_{G W}\right)^{\frac{1}{2}}$ in the plane EMW case, but $N_{x}^{(1)}$ in our case and $2\left(\dot{N}_{0} \dot{N}_{g w}\right)^{1 / 2}$ in the plane EM case have a very different physical behavior) must be effectively larger than the noise photon fluctuation $\left(N_{x}^{(0)} \Delta t\right)^{1 / 2}$, i.e.,

$$
N_{x}^{(1)} \Delta t>\left(N_{x}^{(0)} \Delta t\right)^{1 / 2}
$$

then

$$
\Delta t>N_{x}^{(0)} /\left(N_{x}^{(1)}\right)^{2}=\Delta t_{\min }
$$

where $\Delta t_{\min }$ is requisite minimal signal accumulation time at the noise background $N_{x}^{(0)}$. In fact, Eqs. (50) and (51) are the exact forms from the general relation Eqs. (25), (26), while Eq.(23) is the exact form from the general relation Eqs. (25) (26) in the plane EMW case. In the following we list the $N_{x}^{(1)}, N_{x}^{(0)}, \Delta t_{\min }$ and measurable HFGW strength $h_{r m s}$ at the different receiving surfaces. If $\mathrm{x}=0$ (the yz-plane), then $N_{x}^{(0)}=0$, it would be best measuring in the region for $N_{x}^{(1)}$. Of course, this does not mean that there are no other noise photon fluxes passing through the receiving surface $\Delta s$. In fact, scattering, diffraction and drift of the BPF and the thermal noise caused by the BPF all can generate smaller the noise photon fluxes passing through the surface $\Delta s$. Since they are all caused by the BPF, they should have the same decay factor $\exp \left(-\frac{2 x^{2}}{W^{2}}\right)$ as the BPF. Moreover, external EM noise and the thermal noise caused by the environmental temperature are independent of the BPF, but they can be effectively suppressed by high-quality Faraday cage or shielding covers and low-temperature ( $\mathrm{T} \sim 1 \mathrm{~K}$ or less) vacuum operation. In general, they are much less than the BPF. Issues such as the thermal noise, the radiation press noise, and the noise caused by the scattering for this scheme have been discussed in Ref.[41], we sall not repeat them here. Thus, our attention will be focused only on the BPF itself and the other noise photon flux $N_{x(\text { other })}^{(0)}$ caused by the BPF. In this case, if such noise photon fluxes passing through the receiving surface $\Delta s$ at the yz-plane can be limited a realizable level, then we can estimate the minimal signal accumulation time $\Delta t_{\min }$ in the noise background.

From the above discussion, Eqs.(48), (49) and Ref.[32,40], the signal photon flux $N_{x}^{(1)}$ and the background photon flux $N_{x}^{(0)}$ passing through $\Delta s$ 
are

$$
\begin{gathered}
N_{x}^{(1)}=\left|N_{x}^{(1)}\right|_{\max } \exp \left(-\frac{x^{2}}{W^{2}}\right), \\
N_{x}^{(0)}=\left|N_{x}^{(0)}\right|_{\max } x \exp \left(-\frac{2 x^{2}}{W^{2}}\right),
\end{gathered}
$$

and

$$
N_{x(\text { other })}^{(0)}=\left|N_{x(\text { other })}^{(0)}\right| \max \exp \left(-\frac{2 x^{2}}{W^{2}}\right),
$$

Displaying condition in the receiving surfaces will be

$$
N_{x}^{(1)}(\Delta t)^{\frac{1}{2}} \geq\left[N_{x}^{(0)}+N_{x(\text { other })}^{(0)}\right]^{\frac{1}{2}},
$$

thus

$$
\Delta t \geq \frac{x\left|N_{x}^{(0)}\right|_{\max }+\left|N_{x(\text { other })}^{(0)}\right|_{\max }}{\left|N_{x}^{(1)}\right|_{\max }^{2}} \text { and } \Delta t_{\min }=\frac{x\left|N_{x}^{(0)}\right|_{\max }+\left|N_{x(\text { other })}^{(0)}\right|_{\max }}{\left|N_{x}^{(1)}\right|_{\max }^{2}}
$$

where $\left|N_{x}^{(0)}\right|_{\max } \approx 1.2 \times 10^{22} s^{-1}$ in the typical parameters condition of the scheme.

Considering a possible laboratory condition, we choice the typical parameters in Ref.[32], i.e., $\hat{B}_{y}^{(0)}=3 T, L=6 m, P=10 \mathrm{~W}$. Then we can estimate $\Delta t_{\min }$ in the different HFGW parameters conditions.

(1) $\mathrm{x}=0$, then $N_{x}^{(0)} \equiv 0$, from Eqs. (53) and (56)

$$
\Delta t_{\min }=\frac{\left|N_{x(\text { other })}^{(0)}\right|_{\max }}{\left|N_{x}^{(1)}\right|_{\max }^{2}} .
$$

$$
\begin{aligned}
& \text { If } \hat{h}=10^{-30}, \text { then } N_{x}^{(1)}=\left|N_{x}^{(1)}\right|_{\max } \approx 8.2 \times 10^{2} \mathrm{~s}^{-1} \text { and } \\
& \Delta t_{\min } \approx 3.0 \times 10^{3} \mathrm{~s} \text { provided } \mid N_{x(\text { other })}^{(\max }<2.1 \times 10^{9} \mathrm{~s}^{-1}, \\
& \Delta t_{\min } \approx 3.0 \times 10^{5} \mathrm{~s} \sim 3.5 \text { days provided }\left|N_{x(\text { other })}^{(0)}\right|_{\max }<2.1 \times 10^{11} \mathrm{~s}^{-1} . \\
& (\sim 0.7 \mathrm{PW})
\end{aligned}
$$




$$
\begin{aligned}
& \hat{h}=10^{-27}, \text { then }\left|N_{x}^{(1)}\right|_{\max } \approx 8.2 \times 10^{5} \mathrm{~s}^{-1} \text { and } \\
& \Delta t_{\min } \approx 3.0 \times 10^{3} s \text { provided }\left|N_{x(\text { other })}^{(0)}\right|_{\max }<2.1 \times 10^{15} \mathrm{~s}^{-1}, \\
& \Delta t_{\min } \approx 3.0 \times 10^{5} s \sim 3.5 \text { days provided }\left|N_{x(\text { other })}^{(0)}\right|_{\max }<2.1 \times 10^{17} \mathrm{~s}^{-1} \text {. } \\
& \hat{h}=10^{-26}, \text { then }\left|N_{x}^{(1)}\right|_{\max } \approx 8.2 \times 10^{6} \mathrm{~s}^{-1} \text { and } \\
& \Delta t_{\min } \approx 3.0 \times 10^{3} s \text { provided }\left|N_{x(\text { other })}^{(0)}\right|_{\max }<2.1 \times 10^{17} \mathrm{~s}^{-1}, \\
& \Delta t_{\min } \approx 3.0 \times 10^{5} s \sim 3.5 \text { days provided } N_{x(\text { other })}^{(0)}<2.1 \times 10^{19} \mathrm{~s}^{-1} . \\
& \hat{h}=10^{-24}, \text { then }\left|N_{x}^{(1)}\right|_{\max } \approx 8.2 \times 10^{8} \mathrm{~s}^{-1} \text { and } \\
& \Delta t_{\min } \approx 3.0 \times 10^{3} s \text { provided }\left|N_{x(\text { other })}^{(0)}\right|_{\max }<2.1 \times 10^{21} \mathrm{~s}^{-1}, \\
& \Delta t_{\min } \approx 3.0 \times 10^{5} s \sim 3.5 \text { days provided }\left|N_{x(\text { other })}^{(0)}\right|_{\max }<2.1 \times 10^{23} \mathrm{~s}^{-1} .
\end{aligned}
$$

The above results show that limitation to the other noise photon fluxes passing through $\Delta s$ would be very relaxed. It is interesting to compare the scheme employed earlier (see Eq. (23), where $h=10^{-26}, v=$ $5 G H z, \hat{B}_{y}^{(0)}=10 T, L=10 m, P=10 \mathrm{~W}$ ) and the current scheme (see Eq.(60), here $\left.\hat{h}=10^{-26}, v=5 G H z, \hat{B}^{(0)}=3 T, L=6 m, P=10 \mathrm{~W}\right)$, they show that the current scheme has obvious advantages and reality.

(2) $\mathrm{x}=1 \mathrm{~cm}=10^{-2} \mathrm{~m}$, then $N_{x}^{(0)} \approx 1.1 \times 10^{20} \mathrm{~s}^{-1}$, but where $\left|N_{\left.x_{(\text {other }}\right)}^{(0)}\right|_{\max }$ is often much less than $N_{x}^{(0)}$ i.e., $N_{x_{(o t h e r)}}^{(0)}$ can be neglected in the all following discussions. From Eq.(56), we have

$$
\begin{aligned}
& \hat{h}=10^{-26}, \quad N_{x}^{(1)} \approx 7.8 \times 10^{6} s^{-1}, \quad \Delta t_{\min } \approx 1.8 \times 10^{6} s . \\
& \hat{h}=10^{-25}, \quad N_{x}^{(1)} \approx 7.8 \times 10^{7} s^{-1}, \quad \Delta t_{\min } \approx 1.8 \times 10^{4} s . \\
& \hat{h}=10^{-24}, \quad N_{x}^{(1)} \approx 7.8 \times 10^{8} s^{-1}, \quad \Delta t_{\min } \approx 1.8 \times 10^{2} s .
\end{aligned}
$$

(3) $x=2 \mathrm{~cm}=2 \times 10^{-2} \mathrm{~m}$, then $N_{x}^{(0)} \approx 1.7 \times 10^{20} \mathrm{~s}^{-1}$

$$
\begin{aligned}
& \hat{h}=10^{-26}, \quad N_{x}^{(1)} \approx 7.0 \times 10^{6} \mathrm{~s}^{-1}, \quad \Delta t_{\min } \approx 3.5 \times 10^{6} s . \\
& \hat{h}=10^{-25}, \quad N_{x}^{(1)} \approx 7.0 \times 10^{7} \mathrm{~s}^{-1}, \quad \Delta t_{\min } \approx 3.5 \times 10^{4} s . \\
& \hat{h}=10^{-24}, \quad N_{x}^{(1)} \approx 7.0 \times 10^{8} \mathrm{~s}^{-1}, \quad \Delta t_{\min } \approx 3.5 \times 10^{2} s .
\end{aligned}
$$


(4) $x=3 \mathrm{~cm}=3 \times 10^{-2} \mathrm{~m}$, then $N_{x}^{(0)} \approx 1.8 \times 10^{20} \mathrm{~s}^{-1}$

$$
\begin{aligned}
& \hat{h}=10^{-26}, \quad N_{x}^{(1)} \approx 5.8 \times 10^{6} \mathrm{~s}^{-1}, \quad \Delta t_{\min } \approx 5.4 \times 10^{6} s . \\
& \hat{h}=10^{-25}, \quad N_{x}^{(1)} \approx 5.8 \times 10^{7} \mathrm{~s}^{-1}, \quad \Delta t_{\min } \approx 5.4 \times 10^{4} s . \\
& \hat{h}=10^{-24}, \quad N_{x}^{(1)} \approx 5.8 \times 10^{8} \mathrm{~s}^{-1}, \quad \Delta t_{\min } \approx 5.4 \times 10^{2} s .
\end{aligned}
$$

(5) $x=10 \mathrm{~cm}=0.1 \mathrm{~m}, N_{x}^{(0)} \approx 4.0 \times 10^{17} \mathrm{~s}^{-1}$

$$
\begin{aligned}
& \hat{h}=10^{-26}, \quad N_{x}^{(1)} \approx 1.5 \times 10^{5} s^{-1}, \quad \Delta t_{\min } \approx 1.2 \times 10^{7} s . \\
& \hat{h}=10^{-25}, \quad N_{x}^{(1)} \approx 1.5 \times 10^{6} s^{-1}, \quad \Delta t_{\min } \approx 1.2 \times 10^{5} s . \\
& \hat{h}=10^{-24}, \quad N_{x}^{(1)} \approx 1.5 \times 10^{7} s^{-1}, \quad \Delta t_{\min } \approx 1.2 \times 10^{3} s .
\end{aligned}
$$

(6) $x=29 \mathrm{~cm}$, (about distance of 6 spot radiuses of the GB),

$\hat{h}=10^{-26}$, then $N_{x}^{(0)} \approx N_{x}^{(1)} \approx 2.1 \times 10^{-8} \mathrm{~s}^{-1}$. Time of

receiving one transversal photon would be $\Delta t_{\min } \approx \frac{1}{N_{x}^{(0)}} \approx \frac{1}{N_{x}^{(1)}}$

$\approx \frac{1}{2.1 \times 10^{-8} \mathrm{~s}^{-1}}=4.8 \times 10^{7} \mathrm{~s}$.

The above numerical estimation shows that:

1. The best position for displaying $N_{x}^{(1)}$ would be in the yz-plane and the other parallel receiving surfaces in the region of $-2 \mathrm{~cm}<x<$ $2 \mathrm{~cm}$. In such regions, the transverse PPF $N_{x}^{(1)}$ for the parameter condition $\hat{h} \sim 10^{-24}-10^{-30}$ may reach up to $\sim 8.2 \times 10^{8} s^{-1}$ to $8.2 \times$ $10^{2} \mathrm{~s}^{-1}$. If other noise photon fluxes passing through the surfaces can be effectively suppressed into $\sim 2.1 \times 10^{23} \mathrm{~s}^{-1}$ to $\sim 2.1 \times 10^{9} \mathrm{~s}^{-1}$, then corresponding minimal signal accumulation time $\Delta t_{\min }$ in the noise photon flux background would be $\sim 10^{3} \mathrm{~s}$ to $10^{5} \mathrm{~s}$.

2. Unlike $N_{x}^{(1)}, N_{x}^{(0)}$ has maximum at $x \sim 3.2 \mathrm{~cm}$, where $N_{x}^{(0)} \gg N_{x}^{(1)}$, but $\left.N_{x}^{(1)}\right|_{x=3.2 \mathrm{~cm}}$ and $\left.N_{x}^{(1)}\right|_{x=0}=\left.N_{x}^{(1)}\right|_{\max }$ have the same order of magnitude. In the region, the detecting sensitivity would be worse by 3-4 orders of magnitude over that at the yz-plane.

3. Since $N_{x}^{(1)}=\left|N_{x}^{(1)}\right|_{\max } \exp \left(-\frac{x^{2}}{W^{2}}\right), N_{x}^{(0)}=\left|N_{x}^{(0)}\right|_{\max } x \exp \left(-\frac{2 x^{2}}{W^{2}}\right)$, even if $\hat{h}=10^{-26}$, they will have the same order of magnitude in $x \approx 29 \mathrm{~cm}$. However, where $N_{x}^{(0)}, N_{x}^{(1)}$ all decay to $2.1 \times 10^{-8} \mathrm{~s}^{-1}$.

Moreover, it was shown that if the propagating detections of $N_{x}^{(0)}$ and $N_{x}^{(1)}$ are the same in $1 \mathrm{st}$, 3rd, 6th and 8th octants in our case, then they will propagate along the opposite directions in the $2 \mathrm{nd}, 4$ th, 5 the and 
7the octants [32]. This means the distinguishing ability to $N_{x}^{(0)}$ and $N_{x}^{(1)}$ of the scheme can be further improved. Also, as suggested by Baker [42], since the BPF is unaffected by the magnetic field (it is only involved in the generation of the PPF), one can differentiate the PPF from the BPF by modulating the magnetic field. This essentially eliminates the BPF by microwave-receiver signal processing. For example, one measures the BPF plus PPF with the magnet on and then measures the BPF alone with the magnet off and subtracts one from the other in order to obtain the PPF alone. This process is accomplished more rigorously by statistical signal processing.

\subsection{Role of fractal membranes or other equivalent microwave lenses}

(1). The FMs is merely one of many possible ways to improve the SNR and detecting quality via the redirection of signal photons onto the microwave detectors [32]. However, in the above discussion, the proposal scheme did not involve the FMs. In order words, even if we do not use the FMs, the above-mentioned relation between the PPF and the BPF is still valid. The fractal membranes in the $\mathrm{GHz}$ band have successfully been developed by the Hong Kong University of Science and Technology [43-45] from 2002-2005. Firstly, the fractal membranes (FMs) have very good selection ability to the photon fluxes in the $\mathrm{GHz}$ band. If the FM is nearly totally reflecting for the photon fluxes with certain frequencies in the $\mathrm{GHz}$ band, then it will be nearly total transmitting for the photon fluxes with other frequencies in the GHz band. Secondly, the FMs have good focus function to the photon fluxes in the $\mathrm{GHz}$ band. For example, the photon fluxes reflected and transmitted by the FMs can keep their strength invariant within the distance of 1 meter from the FMs. Such function has been proven by experimental tests. The role of the FMs in the scheme is only the reflector or the transmitter for the photon flux in the $\mathrm{GHz}$ band. Because $N_{z}^{(0)}, N_{y}^{(0)}$ and $N_{x}^{(0)}, N_{x}^{(1)}$ are exactly orthogonal for each other, an FM (or an equivalent microwave lens) paralle with the yz-plane would focuses only $N_{x}^{(0)}, N_{x}^{(1)}$ and not $N_{z}^{(0)}, N_{y}^{(0)}$. In fact, here requirement for the FMs is also more relaxed, i.e., it does not require focusing the photon flux onto a micron-sized detector even into a point. In the typical parameter condition of the scheme, if the cross 
section of the focusing photon flux and the image size has the same or close size in the detector ( in distance of $\sim 28 \mathrm{~cm}$, ) then the SNR $N_{x}^{(1)} / N_{x}^{(0)}$ at the receiving surface $\Delta s$ and at the image surface $\Delta s^{\prime}$ would be nearly the same. Moreover, because unfocused $N_{z}^{(0)}, N_{y}^{(0)}$ will be decayed to $10^{-7} \mathrm{~s}^{-1}$ at $\mathrm{x}=29 \mathrm{~cm}$, their influence can be neglected there.

(2). If the FM is just laid at the symmetrical plane (the yz-plane) or at the parallel planes very near the yz-plane (see Figs. 7 and 8), then the wave-fronts of the photon fluxes passing through the receiving surfaces $\Delta s$ at the planes would be the plane or the pseudo-plane, i.e., where it is possible to obtain a better focusing effect. The requirement for the focus in the region would be more relaxed than other regions. This is because such focusing quality depends only on the local interaction of the photon fluxes at the receiving surfaces in the region of $|x| \leq 2 \mathrm{~cm}$. Besides, provided the photon fluxes focused by the FM can keep a plane or pseudo-plane wave-front, then $N_{x}^{(0)}, N_{x}^{(1)}$ focused simultaneously on another surface $\Delta s^{\prime}$ would have the same or nearly the same SNR as with that at $\Delta s$. A unique requirement for $N_{x}^{(1)}$ and $N_{x}^{(0)}$ at $\Delta s^{\prime}$ is that $N_{x}^{(1)}(\Delta t)^{\frac{1}{2}}$ should be larger than $\sqrt{N_{x}^{(0)}}$ in a typical experimental time interval $\Delta t$, and this process does not need an image of high-quality at $\Delta s^{\prime}$. Contrarily, if the FM is laid at an obvious nonsymmetrical plane, then it is difficult to focus the photon fluxes due to the spread property of the GB (see Fig. 9).

(3). The photon fluxes $N_{z}^{(0)}$ and $N_{z}^{(1)}$ in the z-direction have a similar property. However, unlike the relation between $N_{x}^{(0)}$ and $N_{x}^{(1)}, N_{z}^{(0)}$ (noise) is much larger than $N_{z}^{(1)}$ (signal) in the almost of all regions. This is a very important difference between the photon fluxes in such two directions.

(4). A major role of the FM or other equivalent microwave lenses in the scheme is their focusing effect and not their superconductivity, and this does not mean that one can measure only $N_{x}^{(1)}$ ("interference term") and not $N^{(0)}$ (background). Also, it does not mean that $N^{(0)}$ is neglected and $N^{(0)}$ does not reach the photon flux detector. Actually, the FM is immersed in the BPF. Thus the BPF will generate the thermal noise in the FM. However, the BPF itself and the thermal noise photons caused by the BPF in the FM have an essential difference. The former is vector and 
has high directivity; the latter are photons of random thermal motion. Under the low-temperature condition, the latter are much less than the former. In particular, $N_{z}^{(0)}, N_{y}^{(0)}$ of the BPF are exactly parallel to the yzplane and exactly perpendicular to $N_{x}^{(0)}$ and $N_{x}^{(1)}$. Thus $N_{z}^{(0)}$ and $N_{y}^{(0)}$ do not provide any direct contribution to the photon flux passing through the receiving surfaces parallel to the yz plane, nor are they reflected, transmitted or focused by the FMs laying at the receiving surfaces. In other words, the photon flux focused by the FM will be $N_{x}^{(0)}, N_{x}^{(1)}$ and $\operatorname{not} N_{z}^{(0)}, N_{y}^{(0)}$. In this case $N_{x}^{(1)}$ and $N_{x}^{(0)}$ would reach simultaneously the detector, but $N_{x}^{(1)}$ and $N_{x}^{(0)}$ in the different receiving surfaces have the different $\operatorname{ratio} N_{x}^{(1)} / N_{x}^{(0)}$, this is an important difference to the plane EMW case. Therefore, it is always possible to choose a best region and the receiving surface to detect the total photon flux $\left(N_{x}^{(0)}+N_{x}^{(1)}\right)$ which has a good SNR. Furthermore, the $N_{x}^{(0)}$ can be differentiated from the $N_{x}^{(1)}$ by modulating the $\hat{B}_{y}^{(0)}$.

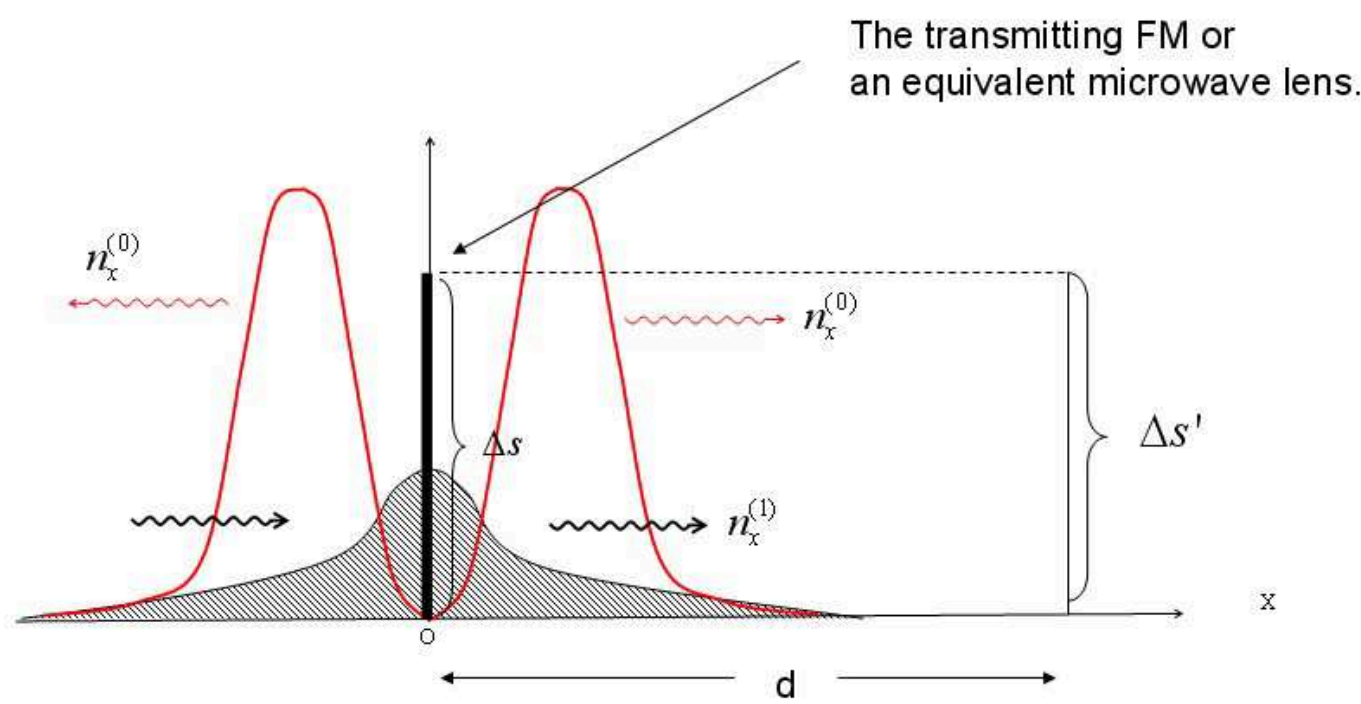

Figure 7: Unlike the photon fluxes $N_{z}^{(0)}, N_{z}^{(1)},\left.N_{x}^{(1)}\right|_{x=0}=\left.N_{x}^{(1)}\right|_{\max }$ where $\left.N_{x}^{(0)}\right|_{x=0}=0$. This means that $N_{x}^{(0)}$ and $N_{x}^{(1)}$ focused by the FM at the yz-plane or at the parallel planes very near the yz-plane would have a good focusing effect and the SNR.

\subsection{Challenge and issues}


The transmitting FM or an equivalent microwave lens

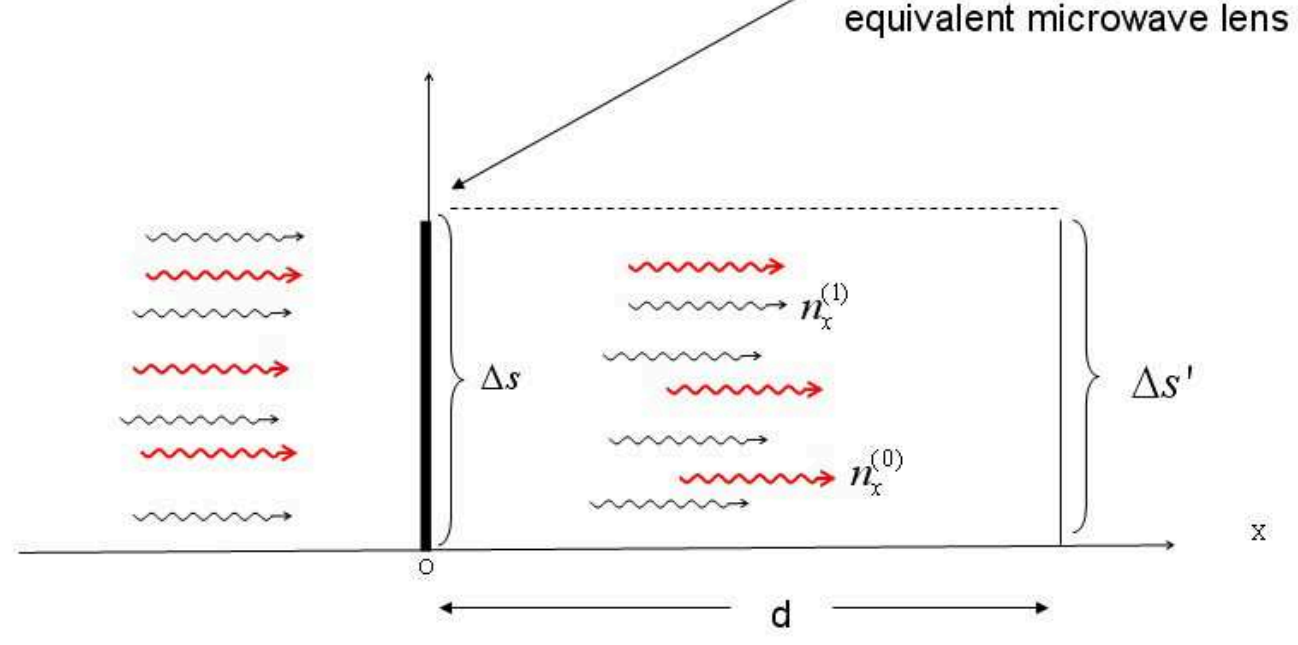

Figure 8: If the FM is just laid at the yz-plane or at the parallel planes very near the yzplane, then the wave-fronts of the photon fluxes passing through the planes would be the plane or the pseudo-plane, and it is possible to obtain an effective focusing effect.

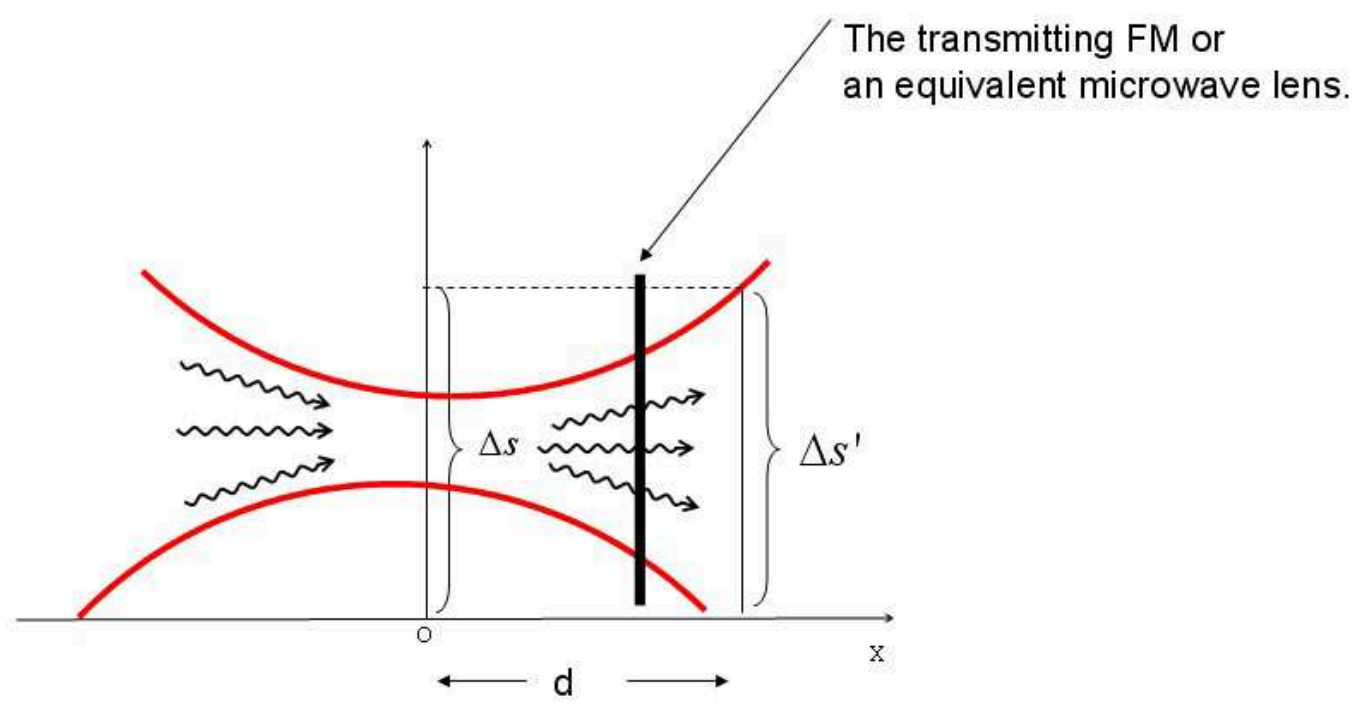

Figure 9: If the FM is laid at an obvious non-symmetrical plane, then it is difficult to focus the photon fluxes due to the spread property of the GB. 
Except for the above-principle analysis, of course, one must consider following challenge and issues. They would include the generation of high-quality GB, suppression of the noises, such as thermal noise, the radiation press noise, noises caused by the scattering of photons, dielectric dissipation due to the dust and other particles, and the concrete influence and correction of the FMs to the GB itself, etc.

The low-temperature ( $\mathrm{T} \sim 1 \mathrm{~K}$ or less) and vacuum operation can effectively reduce the thermal noise and dielectric dissipation. There is room for improvement in other ways as well. They would include utilization of super-strong static magnetic fields, matching of ultra-high sensitivity microwave photon detectors, construction of a good "microwave darkroom", coupling between the open superconducting cavities and the current scheme (the open superconducting cavities have very large quantity factor $\mathrm{Q} \sim 10^{9}-10^{11}$, this coupling might greatly enhance the signal photon flux and not increase obviously the noise power), etc. All these issues need further theoretical study and careful experimental investigation, and they would provide new ways and possibilities to further narrow the gap between the detection schemes and the reality of a valid measurement.

\section{Brief summary}

The EM detecting scheme based on the pure inverse G-effect in the laboratory would not be capable of detecting the HFGWs in the $\mathrm{GHz}$ band, while the coupling system between the Gaussian-type microwave photon flux, the static magnetic field and the fractal membranes (or other equivalent microwave lenses) will be a useful candidate. The key parameter in the current scheme is not the second-order PPF but the transverse first-order PPF; the measurable photon flux is not only the transverse first-order PPF but the total transverse photon flux, and they have different SNRs at the different receiving surfaces; the requisite minimal accumulation time $\Delta t$ of the signal at the special receiving surfaces and in the background photon flux noise would be $\sim 10^{3}-10^{5}$ seconds for the typical laboratory condition and the parameters of $\hat{h} \sim 10^{-26}-10^{-30}$ at $v=5 \mathrm{GHz}$ with bandwidth $\sim 1 \mathrm{~Hz}$

This paper does not involve the standard quantum limit (SQL) caused by the quantum back-action. The SQL constrains the lowest possible sensitivity. We shall show that the SQL in the current scheme does 
not constrain predicated sensitivity (including the constant amplitude HFGWs and the stochastic high-frequency relic GWs). In other words, the sensitivity in the current scheme is the photon signal limited, not quantum noise limited[46]. We will discuss relative issues elsewhere. 


\section{Acknowledgements}

We would like to thank Dr. A. Beckwith for his very useful discussions and suggestion. This work is supported by the National Nature Science Foundation of China under Grant No. 10575140, the Foundation of China Academy of Engineering Physics under Grant No. 2008T0401, 2008T0402, Chongqing University Postgraduates Science and Innovation Fund, Project Number. 200811B1A0100299, GRAVWAVE®LLC, Transportation Science Corporation and Seculine Consulting of the USA. 


\section{References}

[1]R.L.Forward and R.M.L.Baker,' Gravitational gradients, gravitational waves and the 'Weber bar'", Lecture at Lockheed Astrodynansics Research Center, Bel Air,California,650N.Sepulveda,Bel Air,California, USA, November 16th, Lockheed Research Report RL 15210(Forward coined the term "High Frequency Gravitational Waves").(1961).

[2]M.E.Gertsenshtein, Sov.Phys. JETP64,84 (1962)

[3]L.Halpern and B.Laurent,Nuovo Cimento33,728(1964).

[4]R.A.Isaason,Phys.Rev.166,1263(1968)

[5]R.A.Isaason,Phys.Rev.166,1272(1968)

[6]L.P. Grishchuk and M.V.Sazhin,Sov.Phys.JETP32,213(1974)

[7]L.P. Grishchuk and M.V.Sazhin,Sov.Phys.JETP41,787(1975)

[8]G.F. Chapline,J. Nuckolls and L.L.Wood,Phys.Rev.D10,1064(1974)

[9]V.B. Braginsky and V.N. Rudenko, Phys.Reports 46,166(1978)

[10]S.W. Hawking and W. Israd, General Relativity: An Einstein

Centenary Survoy(Cambridge: Cambridge University Press),90-137(1979)

[11]M. Giovannini, Phys. Rev. D 60,123511(1999)

[12]M. Giovannini, Class.Quantum Grav,16,2905(1999)

[13]A. Riagudo and J.P. Ugan,Phys, Rev.D62, 083506(2000)

[14]M, Giovannini, Phy.Rev.D73, 083305(2006)

[15]M,Giovannini,astro-ph/0807(2008)

[16]J.E.Lidsey et al.,Phys,Reports337,343(2000)

[17]M. Gasperini and G. Veneziano, Phys. Reports 373,1(2003)

[18]G.Veneziano,Sci.Am.290,30(2004)

[19]G.S.B.Kogan and V.R.Rudenko, Class Quantum Grav 21,3347(2004)

[20]R.M.L.Baker,R.C.Woods and F.Y.Li,AIP.Proc.813,1280(2006)

[21]P.Chen,Mod.Phys.Lelt.A6,1069(1991)

[22]A.I.Nikishov and V.I.Ritus,Sov. Phys.JETP69,876(1989)

[23]A.I.Nikishov and V.I.Ritus,Sov. Phys.JETP71,643(1990)

[24]G. Gratta et al.,Workshop on Beam-Beam and Beam-Radiation Interaction; High Intensity and Nonlininear Effects,Los Angeles,USA, 1991, edited by C. Pellegrini et al.,(World Scientific,Singapore),70(1992)

[25]P.Chen, Resonant Photon Graviton Conversion in EM Fidas:From

Earth to Heaven, Stanford Linear Accelerator Center-PUB-6666.(September,1994)

[26]X.G.Wu and Z.Y.Fang,Phys.Rev.D78,094002(2008)

[27]A.M.Cruise, Class.Quantum Grav.17,2525(2000)

[28]A.M. Cruise and R.M.J. Ingley, Class, Quanbum Grav.22.S479 (2005)

[29]R. Ballantini et al. gr-qc/0502054(2005) 
[30]R. Ballantini et al., Class.Quantum Grav,20,3505(2003)

[31]A. Nishigawa et al.,Phys.Rev.D77,022002(2008)

[32]F.Y. Li, R.M.L. Baker,Z.Y. Fang, G.V .Stepheson and Z.Y.Chen,Eur.Phys.J.C56,407(2008)

[33] W.K.De Logi and A.R.Mickelson, Phys. Rev. D16, 2915(1977)

[34] A.N. Cillis and D.D. Harari, Phys. Rev. D54, 4757 (1996)

[35] D.Boccaletti et al., Nuovo Cim.B 70, 129(1970)

[36] L.D. Landau and E.M. Lifshitz. The Classical Theory of Fields. (Nauka, Moscow) 1973, PP. 368-370

[37] P.Chen, Phys. Rev. Lett. 74, 634 (1995)

[38] M.Marklund, G. Brodin and P. Dunsby, Astrophys. J. 536,875(2000)

[39] V.De, Sabbata et al., Sov. J. Nucl. Phys. 8, 53-7 (1969)

[40] A. Yariv, Quantum Electronics $2^{\text {nd }}$ ed, (Wiley, New York, 1975), P.110-129

[41]J.Li, F.Y.Li and Y.H. Zhong,Chinese Physics B 18,922(2009)

[42] R. M. L. Baker, Private Communication, June(2008)

[43] W.J.Wen et.al., Phys. Rev. Lett. 89, 223901 (2002)

[44] L.Zhou et al., Appt. Phys. Lett. 82, 1012 (2003)

[45] B. Hou et al., Opt. Express 13, 9149 (2005)

[46] G. V. Stephenson, "The standard quantum limit for the Li-Baker HFGW detector," Proceedings of the Space, Propulsion and Energy Sciences International Forum (SPESIF), 24-27 February, Edited by Glen Robertson. (Paper 023), American Institute of Physics Conference Proceedings, Melville, NY, Vol. 1103, February 2009, pp. 542-547. 